\title{
Numerical simulation and pressure drop prediction of slug flow in oil/gas pipelines
}

\author{
Z. I. Al-Hashimy ${ }^{1}$, H. H. Al-Kayiem ${ }^{1}$, Z. K. Kadhim ${ }^{2}$ \\ \& A. O. Mohmmed ${ }^{1}$ \\ ${ }^{1}$ Mechanical Engineering Department, \\ Universiti Teknologi PETRONAS, Malaysia \\ ${ }^{2}$ Mechanical Engineering Department, College of Engineering, \\ Wasit University, Iraq
}

\begin{abstract}
The sustainable transportation of liquid fuels in a piping system can be interrupted due to slug flow, which causes the severe unsteady loading on pipelines. A feature that is particularly affected by this problem is the oil transportation pipeline, where gas is often combined with the produced oil. In order to fully understand the behavior of such flows, it is imperative to simulate the effective zones along the span of the pipelines. This will allow the designer of the piping system to estimate the required pumping power through the evaluation of the pressure drop in the slug oil/gas flow. This paper reports the oil/gas flow phenomena in a horizontal pipe with a large diameter of $0.16 \mathrm{~m}$, with 3-dimensional, transient, incompressible fluids, utilizing STAR-CCM+ commercial software. The volume of fluid (VOF) model was adopted to track the interface between the two phases. The operational conditions for the cases studied were extracted for the slug zone from the Baker chart. The slug flow was achieved accordingly, which gives us granted validation with the experimental source. The numerical procedure allowed the determination of the pressure drop. Also, the transient behavior of the slug flow was predicted through the tracking of the slug development in the pipe segment. Moreover, the proposed model could be extended to simulate other types of two-phase flow regimes.
\end{abstract}

Keywords: directed mesh, $O \& G$ transport, piping system, slug flow, two-phase flow, VOF model. 


\section{Introduction}

The oil and gas industries continue to grapple with numerous problems associated with fluid transport in pipelines. Millions of man-hours have been spent on research alone to understand the complex behavior of multiphase flows, yet the issue is still a major challenge today. Predictions of multiphase flow domains can be performed nowadays using sophisticated fluid dynamic software packages. In fact, these numerical simulation suites allow engineers to not only analyze the pipeline design but also forecast the productivity of oil/gas systems.

Unstable flows in pipelines may lead to several operational problems that could sometimes prove very costly. A liquid flow with intermittent chunks of slug is usually irregular and, hence, a huge separator may be required to deal with such instabilities. These disturbing conditions arise due to the interaction of liquid waves, which could propagate further to generate hydrodynamic slugs. Nonetheless, from an experimental point of view; it was found that the hydrodynamic slugs can grow out of proportion over time to form a large mass of slugs [1]. Moreover, mechanisms of slug formations and growth are documented problems that, until now, were not properly understood - in fact, this is the core reason why problematic slugs continue to unceasingly manifest in the relevant industries.

Slug flow refers to the phenomenon where liquid-gas two-phase flows are present in horizontal pipelines over a wide range of intermediate flow rates, resulting in undesirable disturbances caused by the actions of liquid and gas plugs, known as slugs. The plug distribution of liquids and gases in slug flows are highly unique but intermittent, simply due to the nature of the terrain, gas/liquid velocity fluctuations, pigging, etc. A slug unit comprises an aerated liquid slug and an accompanying gas burble, restrained within a liquid film of varying thicknesses. The size of the film usually varies from the minimum value at the front of the following slug to the maximum value at the rear of the preceding slug. In consequence, the slug length remains constant along the direction of travel while the pressure drops systematically across the sections of the pipe [2].

The multiphase flow of gases and liquids are common occurrences in many engineering processes such as oil and gas transport, cooling and condensation processes, geothermal production of steam, and the cooling of advance nuclear reactors. Different scientific papers have reported on this process with regards to such flows in tubing with a small diameter of less than $10 \mathrm{~cm}$. Until now, reports of data on large diameter pipes is relatively scarce in literature, despite the fact that a considerable amount of liquids/gases are consistently being pumped through pipelines prior to separation [3].

The sporadic composition of slugs invariably makes the modeling of twophase gas/vapor-liquid rather complex. One definite challenge involves the determination of the flow field geometry. Depending on the nature of the problem, a flow field can adopt various geometric distributions of components, called flow patterns or flow regimes [4]. Moreover, in chemical engineering problems, assessing the distribution of the phases is always assumed to be part of the general 
solution, and phenomenon such as heat/mass transfer, pressure drop, etc. will most certainly be influenced by such a consideration [5].

Flow regime maps for the two-phase flow in a horizontal pipe have been intensively investigated by many researchers. Baker [6] presented a map of twophase flow in a horizontal pipe utilizing various fluids and showed distinct phase's mass fluxes along with corresponding fluid properties such as density and surface tension.

In this paper, we rely on the superficial velocities of slug flow regime implemented by the Baker chart to simulate the slug flow for gas-oil in a horizontal pipe using transient analysis and directed mesh in the commercial Star$\mathrm{CCM}+$ software. So far, no literature has shown the utilization of the direct meshing technique in this software to simulate two phase flow, which is successfully implemented in the present simulation.

\section{Flow pattern maps}

The Baker flow regime map (Fig. 1) shows the boundaries of the various flow pattern regions as functions of the superficial mass velocity of the air phase $G$ and the ratio of superficial mass velocities of the water and air phase L/G; the dimensionless parameters $\lambda$ and $\psi$ were added so that the chart could be used for any gas/oil combination that differs from the standard combination. The standard combination, where both the parameters $\lambda$ and $\psi$ have equal unity, is water and air flow under atmospheric pressure and at room temperature. By taking into account the appropriate values for $\lambda$ and $\psi$, the geometry of two-phase flows with any gas/oil combination at differing pressures and temperatures, can be predicted using the same chart.

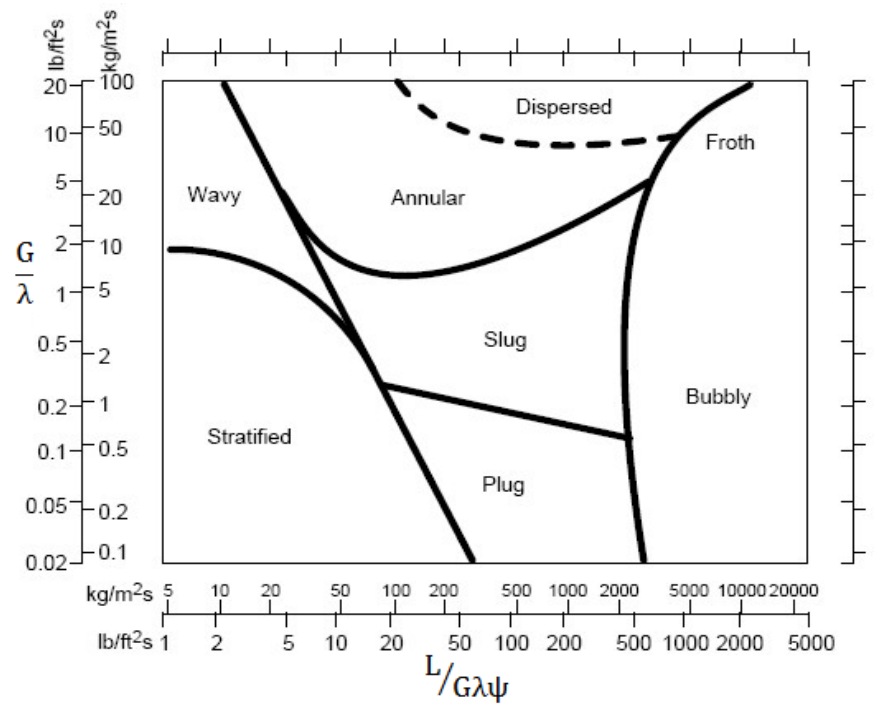

Figure 1: Baker chart [6]. 
Based on the physical properties of oil, gas, water and air, as shown in Table 1, the parameters $\lambda$ and $\psi$ are calculated using eq. (1) and eq. (2), as below:

$$
\begin{gathered}
\Psi=\frac{\sigma_{w}}{\sigma}\left[\left(\mu_{o} / \mu_{w}\right)\left(\rho_{w} / \rho_{o}\right)^{2}\right]^{1 / 3} \\
\lambda=\left[\left(\rho_{g} / \rho_{a}\right)\left(\rho_{o} / \rho_{w}\right)\right]^{0.5}
\end{gathered}
$$

where the subscripts ' $a$ ' and ' $w$ ' refer to air and water, respectively, at normal temperature and atmospheric pressure. The subscripts ' $\mathrm{g}$ ' and 'o' refer to the gas and oil conditions of the simulated fluids.

Table 1: Physical properties of the oil, gas, water and air.

\begin{tabular}{|c|c|c|c|}
\hline Case & $\boldsymbol{\rho}\left[\mathbf{k g} / \mathbf{m}^{\mathbf{3}}\right]$ & $\boldsymbol{\mu}[\mathbf{P a ~ s}]$ & $\boldsymbol{\sigma}[\mathbf{N} / \mathbf{m}]$ \\
\hline Oil & 830 & 0.00332 & 0.019036 \\
\hline Gas & 9.4 & 0.045518 & \\
\hline Water & 998.2 & 0.001003 & 0.07194 \\
\hline Air & 1.225 & 0.045518 & \\
\hline
\end{tabular}

\section{Model development in Star-CCM+}

\subsection{Direct meshing in Star-CCM+}

The horizontal pipe was meshed using a diameter $D_{p}$ of $0.16 \mathrm{~m}$ and a length $L_{p}$ of $87.5 \mathrm{D}_{p}$. The 3D-domain was divided into 105,000 hexahedral cells using directed mesh, where, by using path mesh, we can control the number of grid cells in the cross-section, and using a new volume distribution, we can divide the length of the pipe into any number of grid cells. In this paper, 300 grid cells were used in a cross-section, and 350 grid cells in a longitudinal section, as shown in Fig. 2.

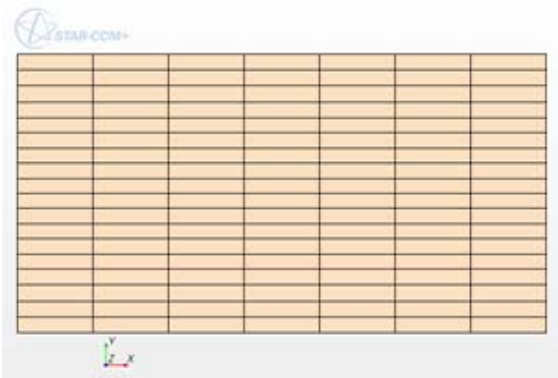

(a)

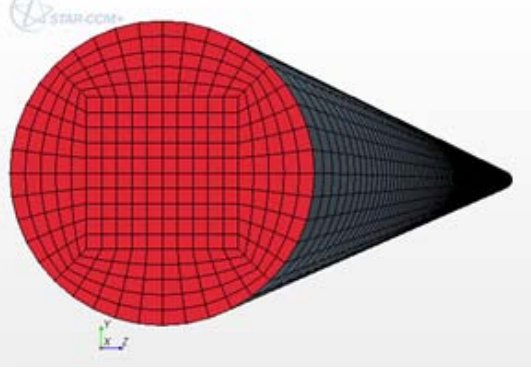

(b)

Figure 2: Directed mesh of the pipe flow with (a) plane section and (b) 3D-domain. 


\subsection{Physical model of slug flow}

The following physics models were specified and used in Star CCM+ code: Three Dimensional, Implicit Unsteady (suitable for any kind of flow), Multiphase Mixture (allows you to specify the bulk properties of the mixture), Eulerian Multiphase model (used to define phases), Multiphase Interaction (used to define phase interactions), Volume of Fluid (VOF), Segregated Flow, The ReynoldsAveraged Navier-Stokes and SST k- $\omega$ (where the turbulent model is selected since it is less computationally demanding and well approximated), All $\mathrm{y}+$ treatment is used to accurately capture the boundary layer near the wall, and Gravity. The primary phase is oil, and the secondary phase is gas. A transient simulation with a time step of $0.001 \mathrm{sec}$ is performed to get a higher degree of temporal accuracy.

\subsection{Boundary conditions}

The boundary conditions are critical components of the Star-CCM+ simulations and it is important that these boundary conditions are specified appropriately. The boundary conditions used in this study are illustrated schematically in Fig. 3.

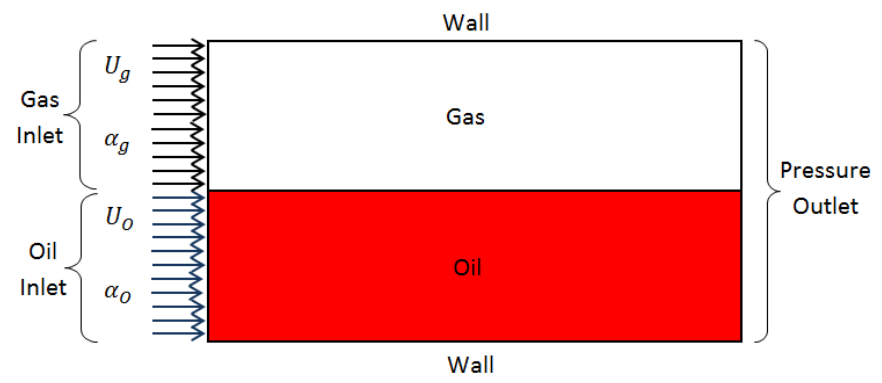

Figure 3: Boundary condition for gas-oil slug flow through a pipe.

Depending on previous studies, there are two methods that were used in the boundary conditions for the simulation of slug flow. The first method was used by Frank [7], where perturbations were imposed at the inlet, so that the volume fraction of liquid phases were entering the pipe as a function of time.

$$
y_{1}=y_{o}+A_{1} \sin \left[2 \pi \frac{U_{o} \cdot t}{p_{1}}\right]
$$

where, $\mathrm{y}_{\mathrm{o}}=0.0, \mathrm{~A}_{1}=0.25 \mathrm{Dp}, \mathrm{p}_{1}=0.25 \mathrm{~L}_{\mathrm{p}}$.

In the second method, the pipe was initially filled with stratified air and water with $50 \%$ volume percentage and zero velocity [8]. For this study the initial and inlet region are the upper half of the pipe, occupied by $50 \%$ volume fraction of the gas phase, $\alpha_{\mathrm{g}}$, and the lower half by $50 \%$ volume fraction of the oil phase, $\alpha_{0}$. Following this, the field function was used to define the inlet oil volume fraction as a function of time and the pressure outlet was imposed to avoid difficulties with backflow at the exit. Based on the Baker chart presented in Fig. 1, the superficial velocities of slug flow regime for gas-oil phases are set as initial and inlet velocity for each phase, as shown in Table 2. 
Table 2: Boundary velocity for the slug flow simulations.

\begin{tabular}{|c|c|c|c|c|c|c|}
\hline Case & $\begin{array}{c}\mathbf{G} / \mathbf{\lambda} \\
{\left[\mathbf{k g} / \mathbf{m}^{2} \mathbf{s}\right]}\end{array}$ & $\begin{array}{c}\mathbf{G} \\
{\left[\mathbf{k g} / \mathbf{m}^{2} \mathbf{s}\right]}\end{array}$ & $\begin{array}{c}\text { Superficial } \\
\text { velocity }[\mathbf{m} / \mathbf{s}]\end{array}$ & $\mathbf{L} / \mathbf{\lambda} \psi \mathbf{G}$ & $\begin{array}{c}\mathbf{L} \\
{\left[\mathbf{k g} / \mathbf{m}^{2} \mathbf{s}\right]}\end{array}$ & $\begin{array}{c}\text { Superficial } \\
\text { velocity }[\mathbf{m} / \mathbf{s}]\end{array}$ \\
\hline Gas-Oil & 10 & 25.3 & 2.691 & 100 & 156.986 & 0.189 \\
\hline
\end{tabular}

\section{Results of gas-oil slug flow}

Slug frequency: The slug period is defined as the time that a slug unit needs to pass through the measuring device. Fig. 4 illustrates the first and last slug along the pipe, where the oil touches the upper half of the pipe and the first oil slug flow appears at $0.63 \mathrm{sec}$, while the last slugs appear at $3.245 \mathrm{sec}$.

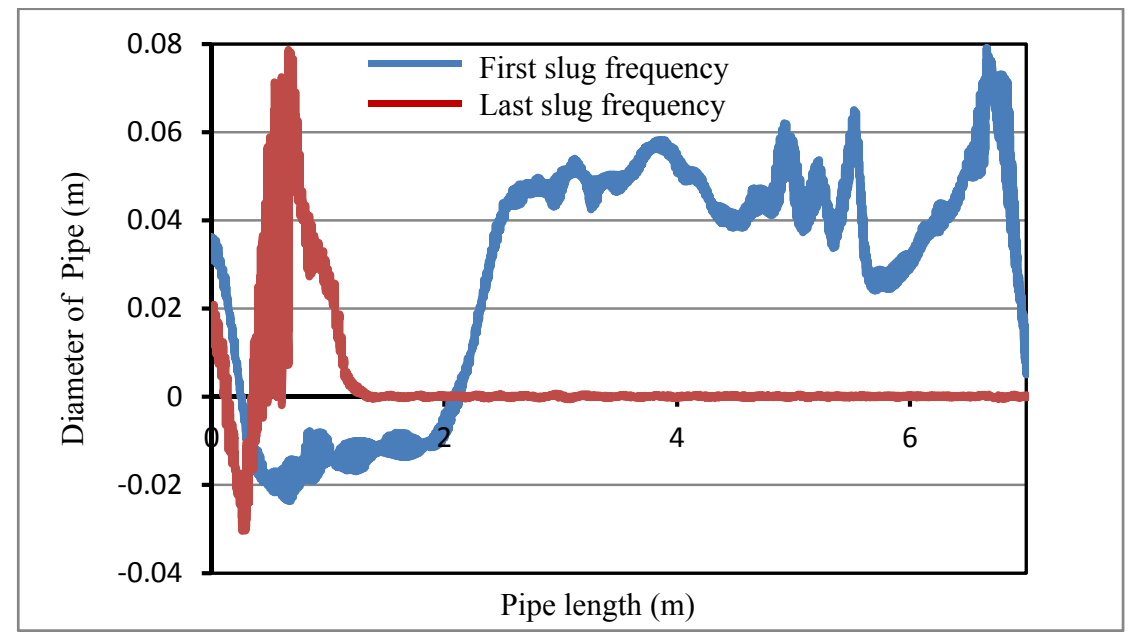

Figure 4: First and last slug frequencies along the pipe.

Volume fraction: The volume fraction in the slug body or gas void fraction is an important parameter for the design of multiphase pipelines and the associated separation equipment. While the phase composition is proportional to its volume fraction, Fig. 5 represent the results of the simulation for a horizontal gas-oil slug flow regime. The distribution of oil and gas in the horizontal flow can be clearly seen. The red color refers to the oil phase, while the dark blue color refers to the gas phase. The best approximation of the slug flow regime is observed when compared with the slug flow regime taken from the Baker chart. Oil slugs are touching the upper part of the pipe and performing complete slug regime.

At the onset, the oil phase was steady until the generation of the first wave crest, which heightened steadily, reaching the cross-section of the pipe (at time $0.753 \mathrm{sec}$ ) and then progressing further along the pipe. The long slug observed (from $0.935 \mathrm{sec}$ to $3.138 \mathrm{sec}$ ), however, was not periodic, as it seemed to have been developed from the initial condition of the flow. 


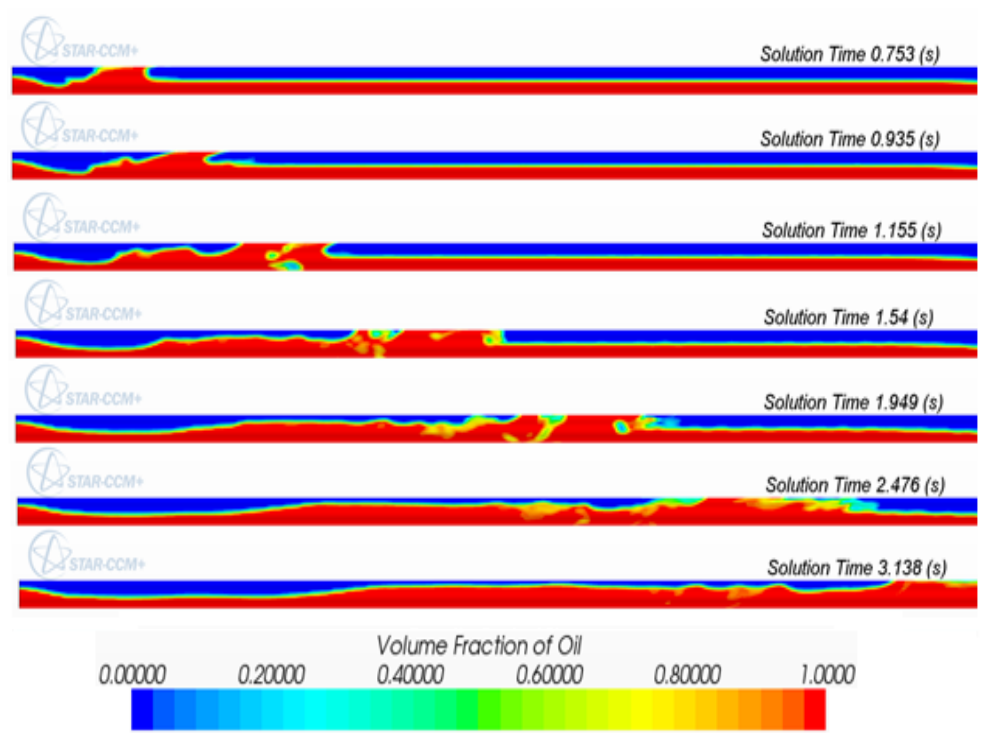

Figure 5: Volume fraction gradients of slug flow for gas-oil.

Pressure drop: Pressure is an important parameter in pipeline design. The pressure loss in a system is an essential variable for the determination of the pumping energy for a given flow. In this study, pressure has been obtained in the form of a time series along the pipe, between the inlet and outlet by using the afield function in Star-CCM+. From Figs 6 and 7, it can be found that the pressure drop for two-phase flow is higher than in a single-phase flow with the same mass flow. A lower density leads to a larger fluid velocity. For the same fluid density, two-phase flow has larger turbulences than a single phase, leading to larger dissipative pressure losses than for a single-phase flow.

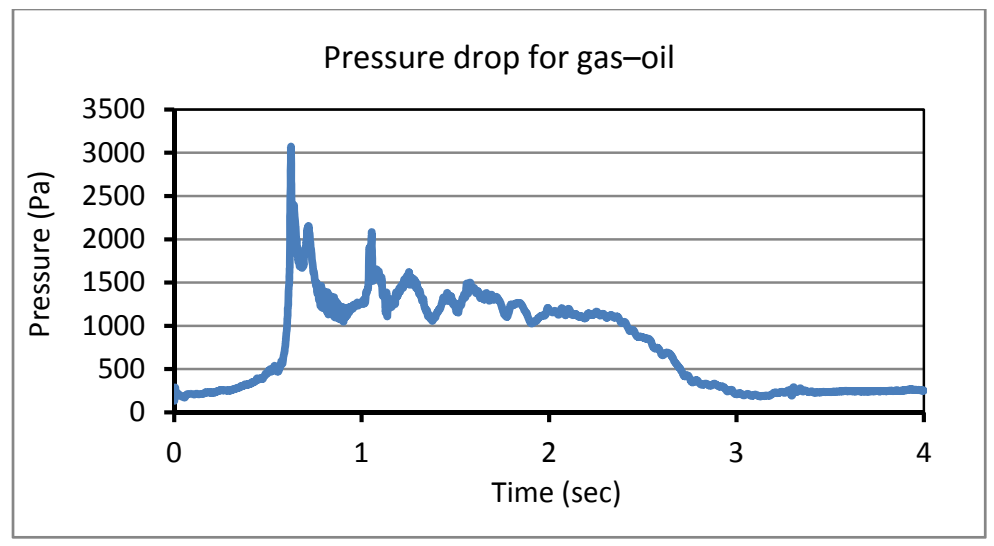

Figure 6: Pressure drop for two-phase oil-gas flow. 


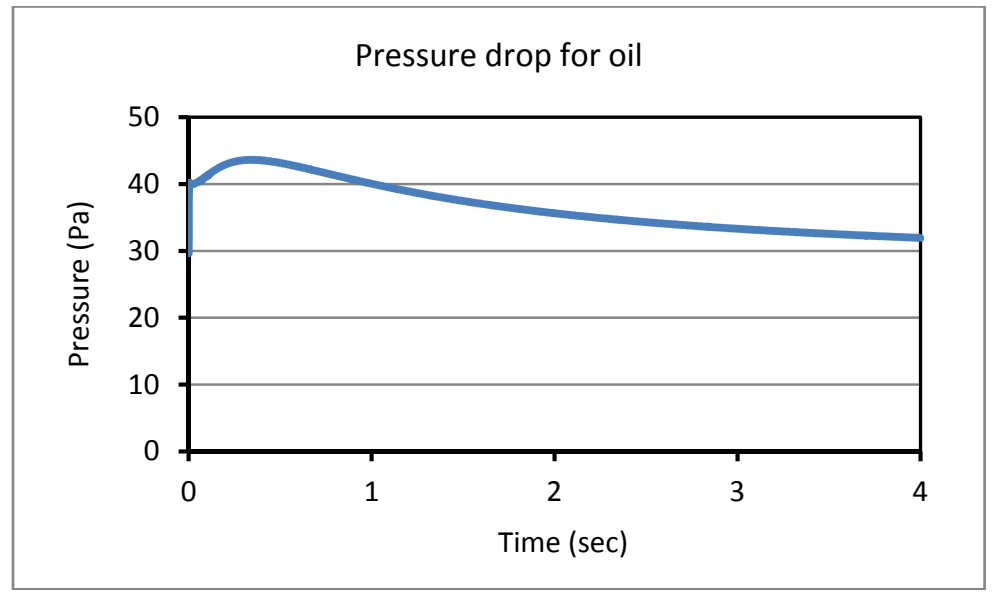

Figure 7: Pressure drop for single phase oil flow.

\section{Conclusions}

The main objective of the presented work was to show the preliminary results of slug flow simulations along a straight pipe, using directed mesh in STAR-CCM+ software.

According to the boundary conditions corresponding to a slug flow regime taken from the Baker chart, a horizontal slug flow regime for oil-gas flow is found.

The directed mesh is suitable to simulate a two-phase flow in a horizontal pipe, where the number of grid cells in the cross-section can be controlled and the length of the pipe can divided into any number of grid cells.

The simulation demonstrates a good agreement with the experimental calculations of a slug flow regime taken from the Baker chart. The property correction factors $\lambda$ and $\psi$ are used for any oil-gas combination and are appropriate for taking the transition from air-water flow to gas-oil flow. The performance of these parameters have been evaluated.

Further investigation into additional simulation results at various operating conditions, and of various pipe inclinations, is recommended.

It should be noted that the highest pressure gets at the first slug frequency. After that, the pressure starts to decline gradually.

\section{Acknowledgement}

The authors acknowledge Universiti Teknologi PETRONAS for sponsoring the research work under the Graduate Assistance scheme (GA). 


\section{References}

[1] Kristiansen, O., Experiments on the transition from stratified to slug flow in multiphase pipe flow. $\mathrm{PhD}$ thesis, Norwegian University of Science and Technology (NTNU), Trondheim, 2004.

[2] Orell, A., Experimental validation of a simple model for gas-liquid slug flow in horizontal pipes. Chemical engineering science 60, no. 5 (2005): 13711381.

[3] Jepson, W. P. \& Taylor, R. E., Slug flow and its transitions in large-diameter horizontal pipes. International journal of multiphase flow 19, no. 3 (1993): 411-420.

[4] Ghorai, S. \& Nigam, K. D. P., CFD modeling of flow profiles and interfacial phenomena in two-phase flow in pipes. Chemical Engineering and Processing: Process Intensification 45, no. 1 (2006): 55-65.

[5] Thome, J. R., Engineering data book III. Wolverine Tube Inc. (2004).

[6] Baker, O., Simultaneous flow of oil and gas, Oil Gas J. 53 (1954): 185-195.

[7] Frank, T., Numerical simulation of slug flow regime for an air-water twophase flow in horizontal pipes. In Proceedings of the 11th International Topical Meeting on Nuclear Reactor Thermal-Hydraulics (NURETH-11), Avignon, France, October, pp. 2-6. 2005.

[8] Mo, S., Ashrafian, A., Barbier, J. C. \& Johansen, S. T., Quasi-3D Modelling of Two-Phase Slug Flow in Pipes. The Journal of Computational Multiphase Flows 6, no. 1 (2014): 1-12. 